\title{
Water interaction with hydrogenated and oxidized detonation nanodiamonds - microscopic and spectroscopic analysis
}

\author{
S. Stehlik ${ }^{1}$, T. Glatzel ${ }^{1}$, V. Pichot $^{2}$, R. Pawlak ${ }^{1}$, E. Meyer ${ }^{1}$, D. Spitzer ${ }^{2}$, B. Rezek $^{3,4}$ \\ ${ }^{1}$ Department of Physics, University of Basel, Klingelbergstrasse 82, 4056 Basel, Switzerland \\ 2 Nanomatériaux pour les Systèmes Sous Sollicitations Extrêmes (NS3E), UMR 3208 \\ ISL/CNRS/Unistra, Institut franco-allemand de recherches de Saint-Louis (ISL), 5, rue du \\ Général Cassagnou, 68300 Saint-Louis, France \\ ${ }^{3}$ Institute of Physics ASCR, Cukrovarnicka 10, 16200 Prague 6, Czech Republic \\ ${ }^{4}$ Faculty of Electrical Engineering, Czech Technical University, Technicka 2, 16627 Prague, \\ Czech Republic
}

stehlik@fzu.cz

\begin{abstract}
Water interaction with surface modified nanodiamonds (NDs) is critical for many possible applications of NDs e.g. in biomedicine. Here we report on investigation of water interaction with hydrogenated and oxidized detonation nanodiamonds (H-DNDs, O-DNDs) by means of Fourier transform infrared spectroscopy (FTIR), thermal analysis, and Kelvin probe force microscopy (KPFM). Higher water content (4.4\%) as well as weaker interaction of water with $\mathrm{H}$ DNDs is identified by thermal analysis. It is explained by hydrophobicity of the H-DNDs surface, as revealed by analysis of bending and stretching vibrations of surface water in FTIR spectra. On the other hand, hydrophilic nature of O-DNDs leads to lower water content $(3.1 \%)$ but stronger interaction with the O-DNDs surface. This is evidenced by as high as $300^{\circ} \mathrm{C}$ desorption temperature of the surface water from O-DNDs. KPFM analysis shows that the surface-bound water can have significant screening effect on contact potential difference (CPD) of nanodiamonds (up to $510 \mathrm{mV}$ ) as well as on the CPD difference between H-DNDs and ODNDs (from $50 \mathrm{mV}$ to $210 \mathrm{mV}$ ). Nevertheless, H-DNDs exhibit always lower work function.
\end{abstract}

Keywords: detonation nanodiamond, surface termination, water, FTIR, KPFM, thermal analysis, contact potential difference, work function

\section{Introduction}

Detonation nanodiamonds (DNDs) represent a fascinating nanomaterial which is made by a detonation of oxygen-deficient explosives such as trinitrotoluene (TNT) and hexogen (RDX). Their size is typically 4-5 nm, however, by using nano-textured explosives one can even further reduce their typical size below $3 \mathrm{~nm}$ as recently shown ${ }^{1,2}$. Once the DNDs are extracted from detonation soot by various wet-chemical purification treatments they can serve in many diverse applications such as polishing, electroplating, lubrication, catalysis, drug delivery, etc. ${ }^{3,4}$ The 
surface properties of DNDs often play an important role due to the high surface-to-volume ratio and the possibility of adjusting the surface chemistry ${ }^{5}$. The most common, well-defined surface chemistry of DNDs is obtained by oxidation or hydrogenation of raw DND powders. Hydrogen termination of DNDs is typically achieved by a hydrogen plasma treatment ${ }^{6,7}$ or by annealing in hydrogen gas at elevated temperature ${ }^{8,9}$. Both processes lead to the formation of carbonhydrogen bonds on the DND surface and the formation of positive zeta potential in colloidal solution of hydrogenated H-DNDs ${ }^{9,}{ }^{10}$. Oxygen termination of DNDs is typically achieved by annealing in air at temperatures between $400-500^{\circ} \mathrm{C}^{11,12}$. In contrast to H-DNDs the formation of functional groups containing oxygen on the surface of DNDs leads to a negative zeta potential of oxidized O-DNDs in colloidal solution ${ }^{10,13}$. It is known that hydrogenated or oxidized surface of bulk diamond (mono or polycrystalline) exhibits very different physicochemical properties such as contact angle, surface conductivity or work function. Whether hydrogenated and oxidized DNDs exhibit also such differences is still an open question ${ }^{14}$ though some recent reports indicate a surface conductivity of $\mathrm{H}$-DNDs ${ }^{15}$ or difference in work function between H-DNDs and O-DNDs ${ }^{10}$.

Geometric constraints of a solid surface as well as the presence of specific functional groups generally leads to structural changes of a surface-bound water layer compared to bulk water properties. In this respect, the water interaction with DNDs is fundamental for both the technology and applications. However it is still not fully understood and explanations of various phenomena are debated. For instance, formation of nanophase water on DNDs was reported, however, no attention was paid to the surface chemistry of the studied DNDs ${ }^{16}$. Recent study showed that orientation of water molecules in the first solvation shell depends on the zeta potential of $\mathrm{NDs}^{17}$. Giant dielectric permittivity of DNDs was also attributed to adsorbed water $(1 \%-4 \%)$ but actual mechanism required further investigation, being tentatively related to protonreleasing functional groups on DNDs ${ }^{18}$.

In this paper we provide experimental evidence of different water interaction with $\mathrm{H}$ DNDs and O-DNDs by using Fourier transform infrared (FTIR) and thermal analysis (TA) techniques. We observe different water adsorption ability and different water binding on $\mathrm{H}$ DNDs and O-DNDs surfaces and we deduce a corresponding structural model. By using Kelvin probe force microscopy (KPFM) we also show how the adsorbed water significantly and differently affects the contact potential difference (CPD) of H-DNDs and O-DNDs that are deposited on highly ordered pyrolitic graphite (HOPG).

\section{Experimental}

Detonation nanodiamonds were synthesized from TNT and RDX precursors in a detonation chamber. The DNDs were purified in $\mathrm{HCl} / \mathrm{HNO}_{3}$ (1:3) and then rinsed with deionised water to $\mathrm{pH} 5^{12}$. Oxidized O-DNDs were obtained by air annealing at $385^{\circ} \mathrm{C}$ for 14 hours. A part of already oxidized O-DNDs was subsequently hydrogenated by annealing under hydrogen atmosphere at $700^{\circ} \mathrm{C}$ for 3 hours. By this approach hydrogenated H-DNDs were obtained. 
The specific surface area (SSA) of H-DNDs and O-DNDs were measured by using an ASAP 2020 system. Thermal desorption at $225^{\circ} \mathrm{C}$ during $12 \mathrm{~h}$ were applied to the samples before measurements. SSA values of $368 \mathrm{~m}^{2} / \mathrm{g}$ and $355 \mathrm{~m}^{2} / \mathrm{g}$ were measured for H-DNDs and ODNDs respectively.

FTIR was measured on a Bruker Tensor 27 apparatus equipped with a single attenuated total reflectance (ATR) accessory (PIKE MIRacle). The detector was a Digitect RT-DLATGS and the resolution was $4 \mathrm{~cm}^{-1}$. The ATR crystal was a diamond (with a $\mathrm{Zn} / \mathrm{Se}$ lens). The measurement was performed at the temperature of $20^{\circ} \mathrm{C}$. The H-DNDs and O-DNDs were measured in form of powders.

TGA was performed on a SII Seiko Instruments Exstar 6000 (TG/DTA 6200) and Differential Scanning Analyses (DSC) was performed using a TA instruments Q1000 apparatus. The experiments were carried out in alumina cups and gold crucibles for TGA and DSC respectively. Both analyses were conducted under a nitrogen flow $(100 \mathrm{~mL} / \mathrm{min})$. An isothermal step at $25^{\circ} \mathrm{C}$ during 2 hours was maintained before heating, and then a ramp of $5^{\circ} \mathrm{C} / \mathrm{min}$ was applied to reach $500^{\circ} \mathrm{C}$.

For the KPFM experiments colloidal suspensions from H-DNDs and O-DNDs powders were prepared at first by ultrasonication (ultrasonic bath 100W) and ultracentrifugation (350000g, $30 \mathrm{~min}$ ), and then used for electrophoretic deposition on highly ordered pyrolytic graphite (HOPG) $)^{19}$ using positive zeta potential of the H-DNDs and negative zeta potential of the O-DNDs. The prepared samples were then left few days at ambient conditions to reach equilibrium with air humidity (typical $\mathrm{RH} \sim 50 \%$ ). The samples were then introduced into a glovebox filled with dry nitrogen, ensuring inert and humidity-free atmosphere $\left(\mathrm{O}_{2}\right.$ and $\mathrm{H}_{2} \mathrm{O}$ were kept below $0.1 \mathrm{ppm}$ level) and analyzed by in-situ KPFM immediately. These KPFM data are denoted as "not annealed". In order to check the effect of adsorbed water on the CPD of HDNDs and O-DNDs an annealing sequence in the glovebox was performed by following steps: $50^{\circ} \mathrm{C}, 100^{\circ} \mathrm{C}, 150^{\circ} \mathrm{C}, 200^{\circ} \mathrm{C}, 250^{\circ} \mathrm{C}, 300^{\circ} \mathrm{C}$ for $30 \mathrm{~min}$, each step followed by immediate $\mathrm{KPFM}$ measurement. A FlexAFM with a C3000 controller (Nanosurf) was used for topography acquisition and a UHFLI lock-in amplifier (Zurich Instruments) served as an external Kelvin controller. The KPFM measurement was carried out in a single pass regime with amplitude (AM) modulation, i.e. both topography and CPD were acquired simultaneously using the first and second resonance of AFM cantilever ${ }^{20}$. For all reported data we used a single Pt/Ir5-coated PPP-EFM cantilever (Nanosensors) with a resonance frequency of $f_{1}=76950 \mathrm{~Hz}$. This fundamental resonance frequency was used for topography acquisition. The second resonance frequency of the cantilever $\left(f_{2}=481498 \mathrm{~Hz}\right)$ was used for KPFM. The free oscillation amplitude was $10 \mathrm{~nm}$, setpoint $65 \%$, scan size $2 \times 2 \mu \mathrm{m}$, scan speed $0.5 \mathrm{~Hz}$, and AC oscillation voltage $1 \mathrm{~V}$. The cantilever was grounded and both $\mathrm{AC}$ oscillation and DC compensation voltage were applied to the sample. Using this arrangement, lower CPD value corresponds to lower work function value however work function of cantilever would need to be determined to get absolute values. We used a compensation of the capacitive crosstalk to obtain accurate KPFM results ${ }^{21}$. 


\section{Results}

Figure 1a shows full range FTIR spectra of DNDs after oxidation in air (O-DNDs, red spectrum) and subsequent hydrogenation (H-DNDs, blue spectrum). The FTIR spectra of both H-DNDs and O-DNDs were acquired several weeks after the respective treatments, stored at ambient conditions meanwhile. Thus we suppose that equilibrium with respect to ambient humidity was reached. The common features in the spectra are related to O-H stretching (2500$\left.3500 \mathrm{~cm}^{-1}\right)$ and bending $\left(1625 \mathrm{~cm}^{-1}\right)$ vibrations from the adsorbed water ${ }^{8,22}$.

In addition, the spectrum of O-DNDs exhibits a carbonyl peak $(\mathrm{C}=\mathrm{O}$ stretching at 1800 $\mathrm{cm}^{-1}$ ) indicating the formation of anhydrides and lactones by air-annealing which shifts the peak to higher wavenumbers than in carboxylic acid groups ${ }^{23}$. A broad absorption feature at 1000$1500 \mathrm{~cm}^{-1}$ is related to a combination of many overlapping peaks, which besides $\mathrm{C}-\mathrm{O}-\mathrm{C}$ stretching may include also the $\mathrm{O}-\mathrm{H}$ deformation and other groups ${ }^{23}$. The FTIR spectrum of $\mathrm{H}-$ DNDs shows the successful hydrogenation by the formation of C-H bonds $\left(2800-3000 \mathrm{~cm}^{-1}\right)$ accompanied by complete disappearance of the carbonyl peak and suppression of the carbonoxygen related features in the $1000-1500 \mathrm{~cm}^{-1}$ region. The features in $2200-2400 \mathrm{~cm}^{-1}$ region are not related to DNDs but arise from software subtraction of atmospheric $\mathrm{CO}_{2}$ absorption.

Figure $1 \mathrm{~b}$ shows a magnified range of $1500-1800 \mathrm{~cm}^{-1}$ where the bending vibrations originating from $\mathrm{OH}$ bonds of both H-DNDs and O-DNDs are visualized in detail. We identified a clear difference between the shape of the $-\mathrm{OH}$ related peak of H-DNDs and O-DNDs. While O-DNDs exhibit a single peak with maximum at $1630 \mathrm{~cm}^{-1}$, the H-DNDs exhibit a broader peak with the maximum at $1640 \mathrm{~cm}^{-1}$ and an apparent shoulder at lower wavenumber. This may indicate different bonding states of the $-\mathrm{OH}$ groups on $\mathrm{H}$-DNDs and O-DNDs surfaces which typically come from the adsorbed water. The spectra of both H-DNDs and O-DNDs also exhibit a shoulder around $1590 \mathrm{~cm}^{-1}$ related to $\mathrm{sp}^{2}$ carbon $^{24}$. 

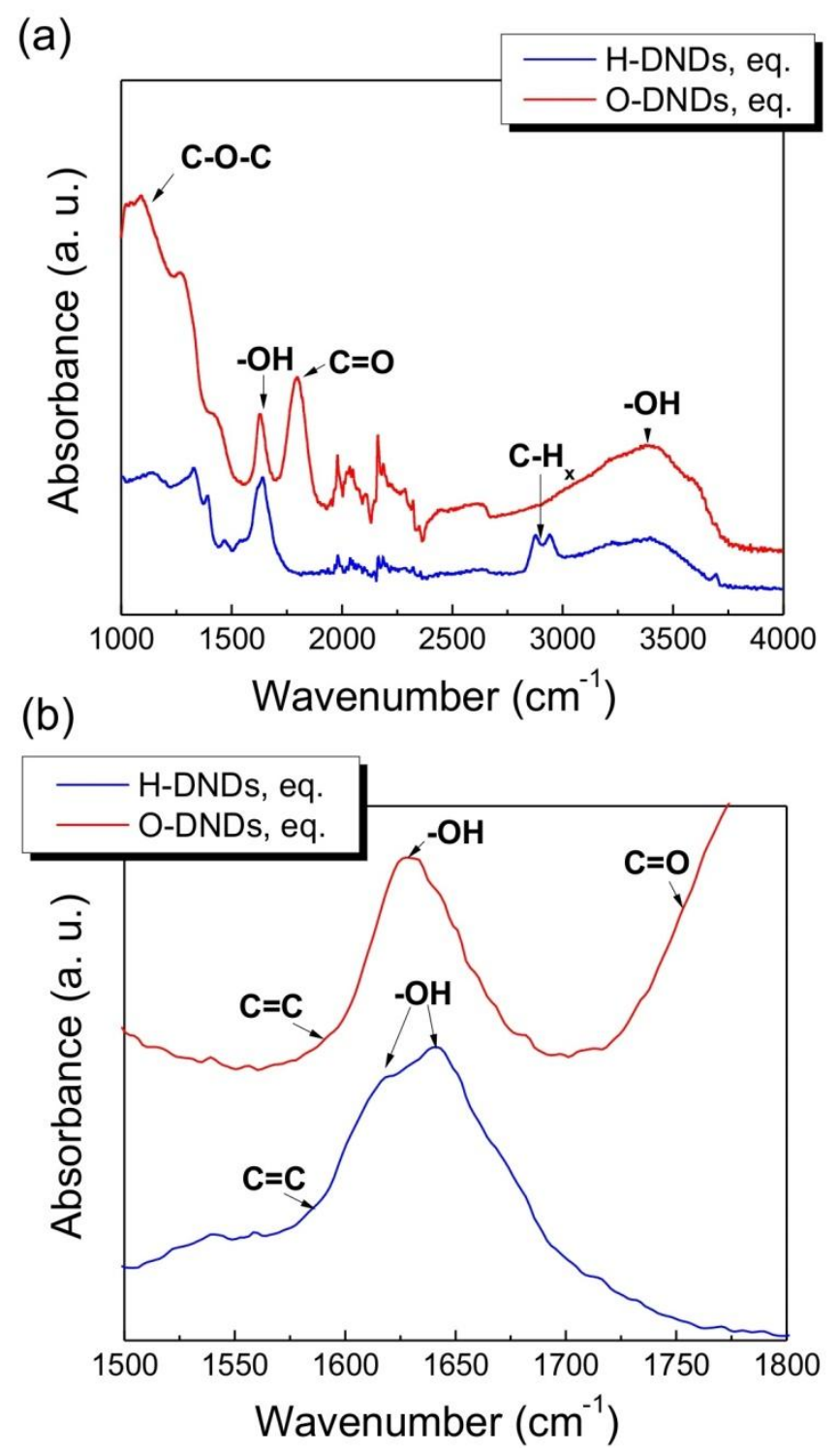

Figure 1. Full range FTIR spectra of H-DNDs (blue curve) and O-DNDs (red curve) equilibrated (eq.) in ambient air (a). Magnified region of 1500-1800 $\mathrm{cm}^{-1}$ shows in detail the different shape of the $-\mathrm{OH}$ bending peak of H-DNDs and O-DNDs (b).

Figure 2a shows the full range FTIR spectra of H-DNDs measured immediately after hydrogenation treatment (black), after several weeks of ambient storage (blue), and after putting them to water and dried again (green). First of all, preservation of the $\mathrm{C}-\mathrm{H}$ bonds shows good long-term stability of such hydrogenated DNDs under various conditions. However, we evidenced the gradual evolution of a $3000-3750 \mathrm{~cm}^{-1}$ broad band coming from the stretching vibrations of $-\mathrm{OH}$ groups which indicates a gradual adsorption of water on the H-DNDs surface. Moreover, a distinct sharp peak at $3690 \mathrm{~cm}^{-1}$ appears with the evolution of the $3000-3750 \mathrm{~cm}^{-1}$ band. There is also an increasing band around $1100 \mathrm{~cm}^{-1}$ in the blue and green spectra in comparison to the freshly hydrogenated DNDs (black). Figure $2 \mathrm{~b}$ shows the $1000-2000 \mathrm{~cm}^{-1}$ 
range in detail. The $-\mathrm{OH}$ related (bending vibrations) peak of freshly hydrogenated H-DNDs (black) clearly exhibits two maxima at $1642 \mathrm{~cm}^{-1}$ and $1616 \mathrm{~cm}^{-1}$. After longer exposure to water from humid air (blue) and liquid water (green) the peak at $1616 \mathrm{~cm}^{-1}$ becomes a shoulder and the peak at $1642 \mathrm{~cm}^{-1}$ becomes dominant.

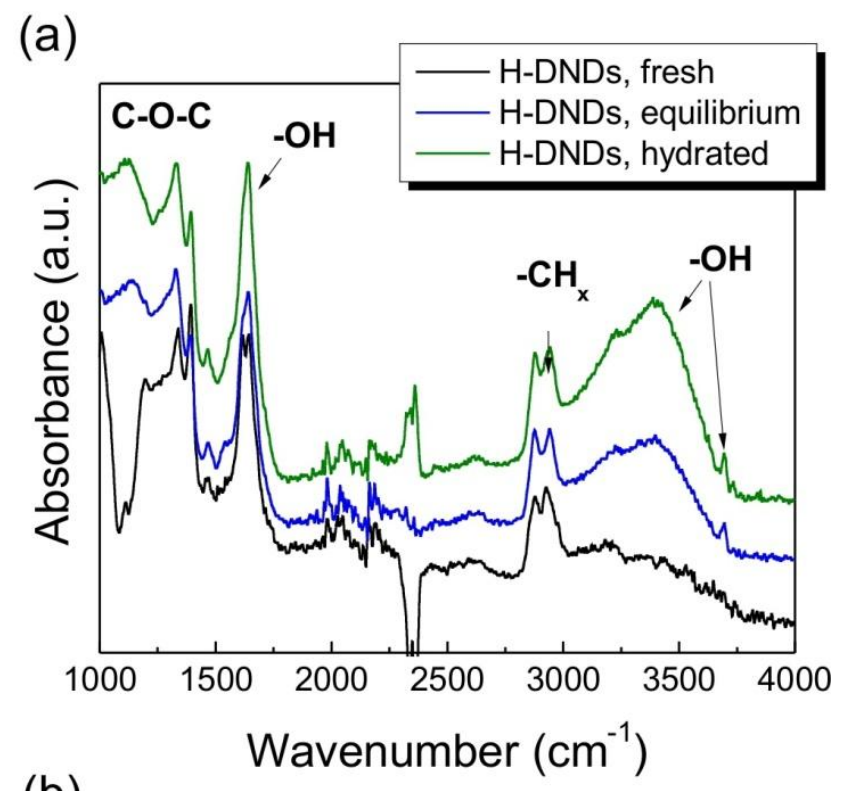

(b)

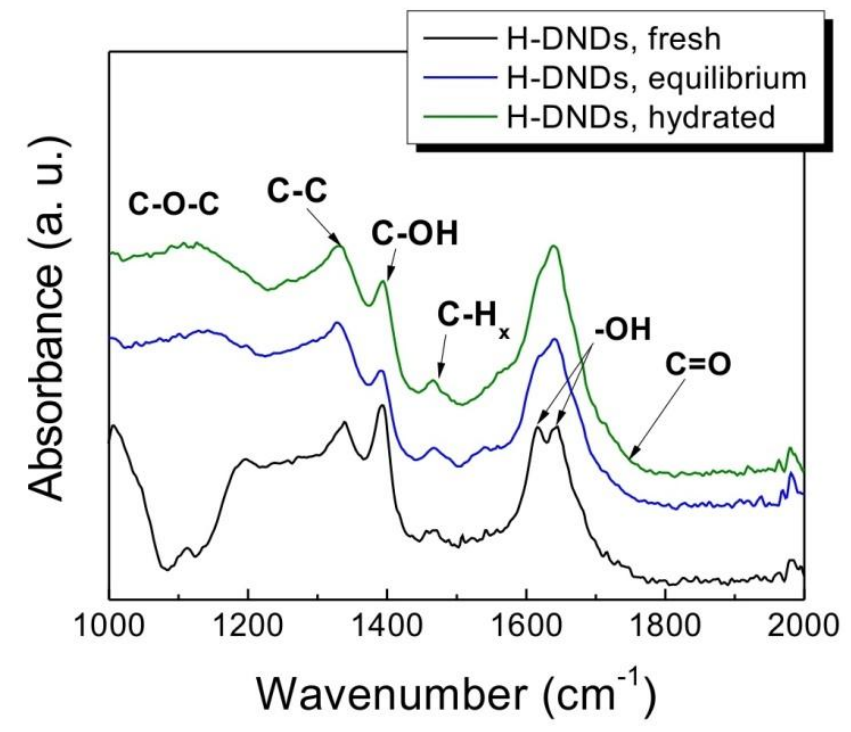

Figure 2. Full range FTIR spectra of H-DNDs measured immediately after hydrogenation treatment (black), after several weeks of ambient storage (blue), and after putting in water and dried (green) (a). Magnified region of 1000-2000 $\mathrm{cm}^{-1}$ shows in detail mainly the evolution of the $-\mathrm{OH}$ bending peak of $\mathrm{H}-\mathrm{DND}$ in dependence on history of the particular sample (b).

TGA and DSC curves of H-DNDs and O-DNDs are shown in the Figure 3. Thermal behavior of both H-DNDs and O-DNDs can be roughly divided into three regions. In the first region $\left(\mathrm{I} ; 25-100^{\circ} \mathrm{C}\right)$ the evaporation of bulk water occurs. In the second region (II; $100-300^{\circ} \mathrm{C}$ ) 
mainly desorption of surface bound water happens. In the third region (III; above $300^{\circ} \mathrm{C}$ ) other exothermic processes like oxidation take place. From the presented data it is obvious that the thermal behavior depends on the particular surface chemistry of the DNDs.

The TGA curve of H-DNDs (Figure 3a) indicates that the total water content in H-DNDs powder is $4.4 \%$ and the surface water desorbs up to $250^{\circ} \mathrm{C}$. This is well documented also by the DSC curve of H-DNDs (Figure 3b). An endothermic peak with a minimum around $65^{\circ} \mathrm{C}$ corresponds to the evaporation of bulk water. In consensus with the related TGA curve desorption of surface bound water continues up to $250^{\circ} \mathrm{C}$. Beyond this temperature a mild increase of the mass occurs as a result of the oxidation of $\mathrm{C}-\mathrm{H}$ bonds by residual oxygen. This oxidation is clearly visible on the DSC curve as two exothermic peaks above $300^{\circ} \mathrm{C}$.

The TGA curve of O-DNDs (Figure 3a) shows that the total water content in O-DNDs in powder form is $3.1 \%$ and the surface water desorbs up to $300^{\circ} \mathrm{C}$ as evidenced by broad endothermic peak on the DSC curve between $100-300^{\circ} \mathrm{C}$. The more rapid mass loss above $400^{\circ} \mathrm{C}$ is most probably related to oxidative etching of the O-DNDs which exhibit itself as an exothermic rise on the DSC curve. H-DNDs are noticeably more resistant to the mass loss, most likely because $\mathrm{C}-\mathrm{H}$ bonds must be oxidized prior to diamond etching. 
(a)

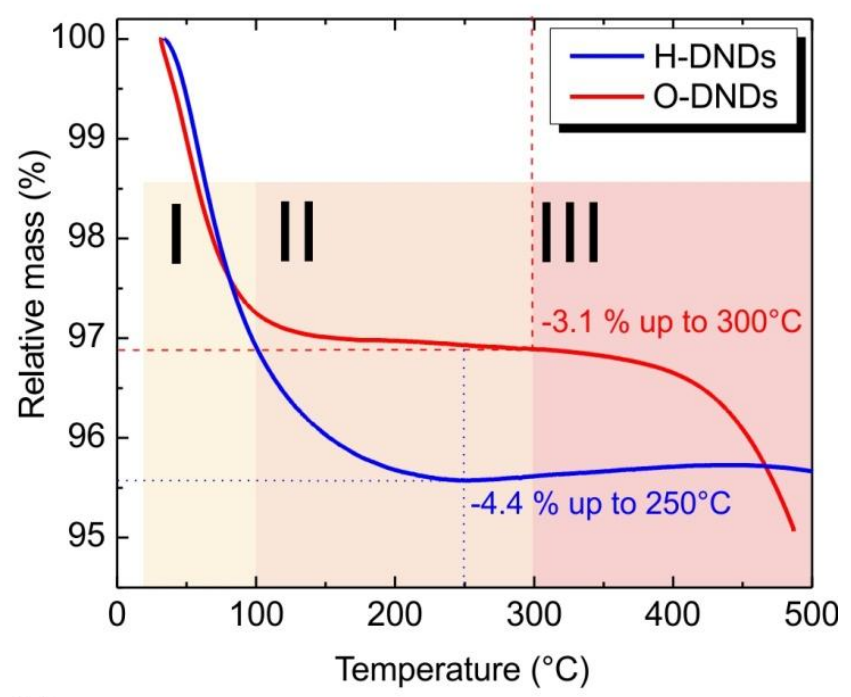

(b)

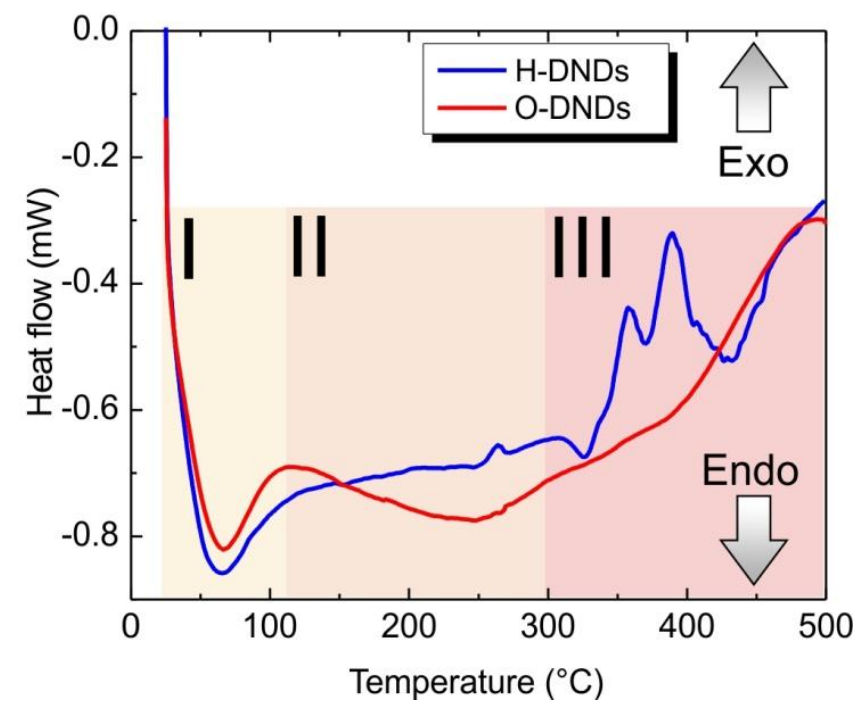

Figure 3. TGA (a) and DSC (b) curves of H-DNDs (blue) and O-DNDs (red).

KPFM images and related CPD histograms in dependence on the annealing temperature are shown in the Figure 4 for both H-DNDs and O-DNDs. The annealing sequence from room temperature up to $300^{\circ} \mathrm{C}$ has significant effect on the CPD of both H-DNDs and O-DNDs while the CPD of HOPG substrate remains almost unchanged. It can thus serve as good reference of CPD and it indicates no significant tip change during the KPFM experiment at the same time providing $\triangle \mathrm{CPD}$ values of the DNDs versus $\mathrm{HOPG}\left(\triangle \mathrm{CPD}=\mathrm{CPD}_{\mathrm{DNDs}}-\mathrm{CPD}_{\mathrm{HOPG}}\right)$. In the case of H-DNDs there is large shift from positive $\triangle$ CPD values prior annealing $(\sim+110 \mathrm{mV})$ down to strongly negative $\triangle \mathrm{CPD}$ values $(\sim-400 \mathrm{mV})$ after the annealing at $300^{\circ} \mathrm{C}$. Although the O-DNDs exhibit a similar trend they have a higher $\triangle \mathrm{CPD}(\sim+160 \mathrm{mV})$ prior to annealing and a lower $\triangle \mathrm{CPD}(\sim-190 \mathrm{mV})$ after the annealing at $300^{\circ} \mathrm{C}$ at variance with the H-DNDs data. KPFM data 
thus show only slight $(\sim 50 \mathrm{mV})$ CPD difference between H-DNDs and O-DNDs before annealing, in agreement with similar prior experiments on gold and silicon ${ }^{10}$ and pronounced CPD difference $(\sim 210 \mathrm{mV})$ after annealing. The KPFM data also show that the annealing in the glovebox has more pronounced effect on H-DNDs than on O-DNDs. This is clearly evidenced by the ratio of H-DND and O-DND $\triangle$ CPD values prior and after annealing: $(160 / 110=1.45)<$ $(400 / 190=2.11)$.
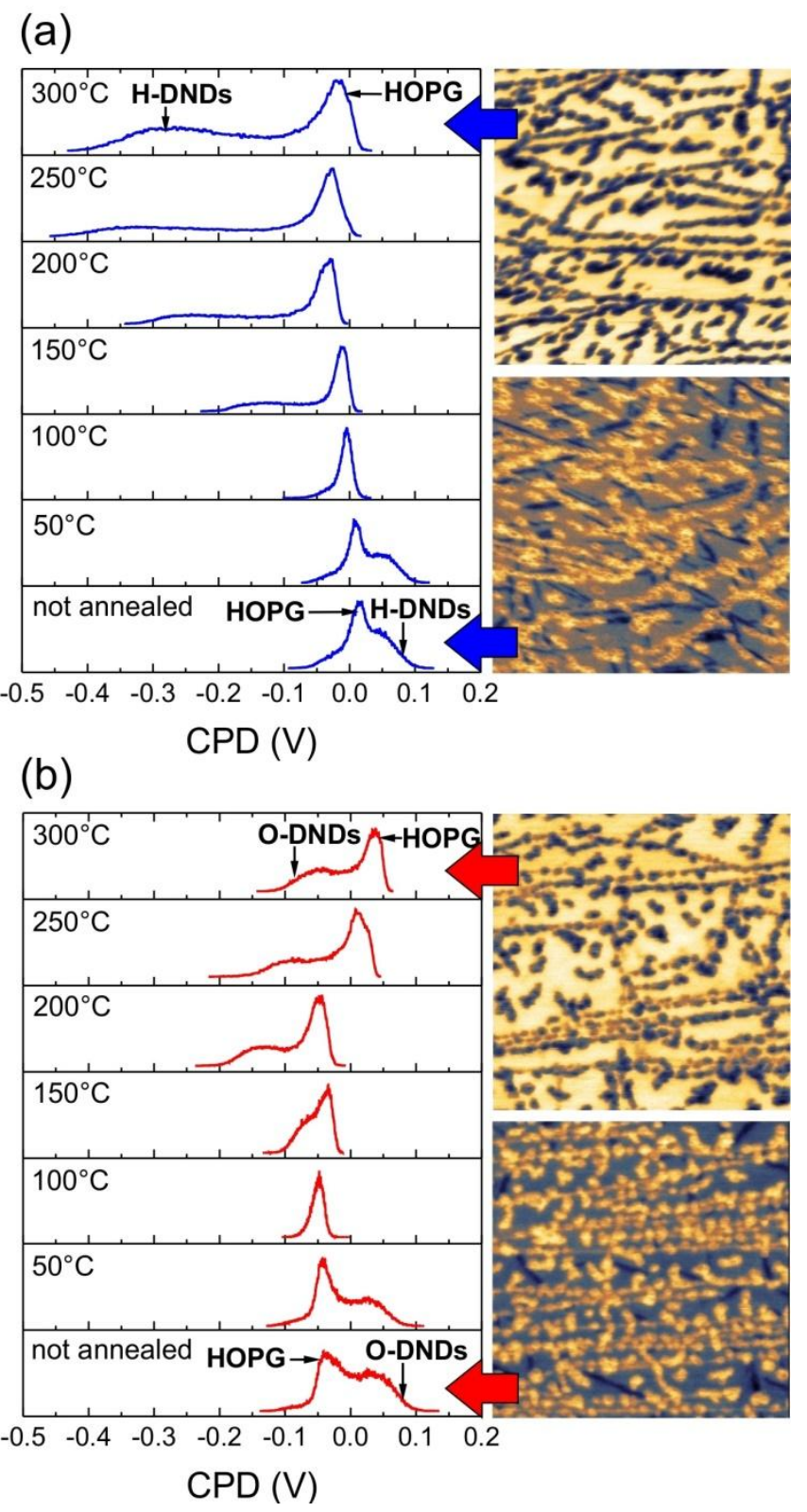

Figure 4. Histograms of the CPD values (H-DNDs (a), O-DNDs (b)) extracted from KPFM images $\left(2 \times 2 \mu \mathrm{m}^{2}\right)$ before and after $30 \mathrm{~min}$ at particular temperature up to $300^{\circ} \mathrm{C}$. Initial and final $\mathrm{KPFM}$ images before and after annealing at $300^{\circ} \mathrm{C}$ are shown for both H-DNDs and O-DNDs, and marked by arrows for easy assignment. 


\section{Discussion}

Our FTIR data show significant differences in the stretching and bending vibrations of $\mathrm{OH}$ bonds between H-DNDs and O-DNDs. Particularly, we identified a distinct sharp peak in the stretching region at $3690 \mathrm{~cm}^{-1}$ which appears only in the spectra of H-DNDs that are saturated with water (Fig.2a eq. and sol.). As all the vibrational bands in liquid water originate from contributions of water molecules in different hydrogen bonded environments, the molecular stretch vibrations shift to higher frequency as hydrogen bonding weakens ${ }^{25}$, whereas the molecular bending vibration peak shifts to lower frequencies and becomes both narrower and stronger $^{26}$. Scatena et al. ${ }^{27}$ showed that the hydrogen bonding between adjacent water molecules at the hydrophobic-water interfaces is weak. This weakening was also theoretically calculated for water on H-terminated bulk diamond surfaces ${ }^{28}$. The weak hydrogen bonding then leads to a strengthening of the $\mathrm{OH}$ oscillator resulting in a blue shift in energy and sharpening of spectral peaks ${ }^{29}$. Moreover, interactions between these water molecules and a hydrophobic surface result in substantial orientation of these weakly hydrogen-bonded water molecules in the interfacial region. The stretching peak at $3690 \mathrm{~cm}^{-1}$ thus corresponds to the "free" $\mathrm{OH}$ bond of water molecules that straddle the interface between the water and hydrophobic surface of H-DNDs. The wavenumber of the "free" $\mathrm{OH}$ bond depends on specific condition at the interface. For instance, $3660 \mathrm{~cm}^{-1}$ for water/propan-1-ol interface ${ }^{30}$ and $3669 \mathrm{~cm}^{-1}$ for water/tetrachloromethane interface ${ }^{27}$. Relatively higher wavenumber found on H-DNDs $\left(3690 \mathrm{~cm}^{-1}\right)$ may be due to rigidity of solid H-DNDs surface where the "free" $\mathrm{OH}$ bond stretching is less damped in contrast to the liquid-liquid interfaces.

The analysis of the bending vibration region of the H-DNDs FTIR spectra (Figure 2b) revealed two distinct peaks at $1616 \mathrm{~cm}^{-1}$ and at $1642 \mathrm{~cm}^{-1}$. The value of bending vibration corresponding to liquid bulk water at $25^{\circ} \mathrm{C}$ is $1643.5 \mathrm{~cm}^{-1}$. We propose that the peak located at $1616 \mathrm{~cm}^{-1}$ originates from initial water interaction with the hydrophobic surface of the H-DNDs and its shift compared to the value of bulk liquid water is a result of the weak hydrogen bond on the H-DNDs-water interface as mentioned above. The second peak at $1642 \mathrm{~cm}^{-1}$ corresponds well with the value of bending vibration for bulk liquid water. This peak as well as the broad $\mathrm{OH}$ stretching $3000-3750 \mathrm{~cm}^{-1}$ feature increase its intensity due to gradual adsorption of "bulk" water. Note, that the strong $-\mathrm{OH}$ bending feature (Figure 2a, maxima at 1616 and $1642 \mathrm{~cm}^{-1}$ ) is already present in the FTIR spectrum of "fresh" H-DNDs (low hydration level) although the $\mathrm{OH}$ stretching feature $\left(2750-3750 \mathrm{~cm}^{-1}\right)$ is negligible and increases only with more pronounced hydration. This may be due to higher absorption cross sections of bending - $\mathrm{OH}$ vibrations in comparison with stretching - $\mathrm{OH}$ vibrations on the surface of H-DNDs.

The bending vibration peak of the O-DNDs has the maximum at $1630 \mathrm{~cm}^{-1}$ (Figure 1b) which suggests generally a weaker hydrogen bond between the water and the O-DNDs surface than in bulk water. The absence of a distinct bulk water peak at $1643.5 \mathrm{~cm}^{-1}$ indicates that most of the water on the O-DNDs is surface-bound water. Mochalin et al. ${ }^{31}$ reported a considerable shift of the bending vibration peak to lower wavenumbers as well as a decrease of its intensity upon 
annealing of acid oxidized DNDs up to $300^{\circ} \mathrm{C}$. Such shift is probably a temperature effect since the higher the temperature the lower is the hydrogen bond strength resulting in a shift to lower frequencies ${ }^{26}$. It is important to mention that according to Mochalin et al. ${ }^{31}$, the bending vibration peak of water disappeared only after 15 min annealing at $425^{\circ} \mathrm{C}$ suggesting rather strong, hydrophilic interaction of water with the surface of oxidized DNDs. Thermal analysis (TGA, DSC) provides complementary information to FTIR about specific the water interaction with H-DNDs and O-DNDs. We showed that H-DNDs can adsorb generally more water than the O-DNDs. This could be explained by the difference in SSA of the H-DNDs compared to ODNDs but we found almost identical SSA of H-DNDs and O-DNDs $\left(\sim 360 \mathrm{~m}^{2} / \mathrm{g}\right)$. Thus we expect that both H-DNDs and O-DNDs have a similar, fractal structure with numerous pores and cavities which is typical for DND powders ${ }^{32,33}$.

The higher water content in the H-DNDs compared to O-DNDs can be thus explained by the formation of a thick water layer on the H-DNDs surface and probably also the formation of water nanodroplets in the porous structure of the powder. Such behavior has been described by the so-called water bridging-cluster model based on thermodynamics that assigns the interaction to organized elongated water clusters between close hydrophobic surfaces ${ }^{34}$. Specific interaction of $\mathrm{H}$-terminated diamond with water has been also indicated by theoretical calculations ${ }^{28}$. Thus it is the different surface chemistry of H-DNDs and O-DNDs and more specifically the hydrophobicity of H-DNDs (similarly to hydrogenated bulk diamond) which causes the higher water content in the H-DNDs. On the other hand, one can expect the formation of a thin water layer on O-DNDs since the oxygen-terminated bulk diamond surface is hydrophilic ${ }^{35}$. It was suggested that an ordered water monolayers may exist on other hydrophilic surfaces like mica ${ }^{36}$ as well as on the oxygen terminated diamond surface ${ }^{37}$. According to the DSC data (Figure 3b) the DSC curve of O-DNDs in the $120-300^{\circ} \mathrm{C}$ region shows a broad endothermic peak which we relate to the desorption of tightly bound surface water.

Based on the FTIR and thermal analysis data and above arguments we suggest the formation of a thick water layer and water nanodroplets in the H-DNDs powder due to hydrophobic interaction of H-DNDs and water. On the other hand, we suggest the predominant formation of a thin water layer which is tightly bound to the hydrophilic surface of O-DNDs. This model is schematically visualized in Figure 5. 

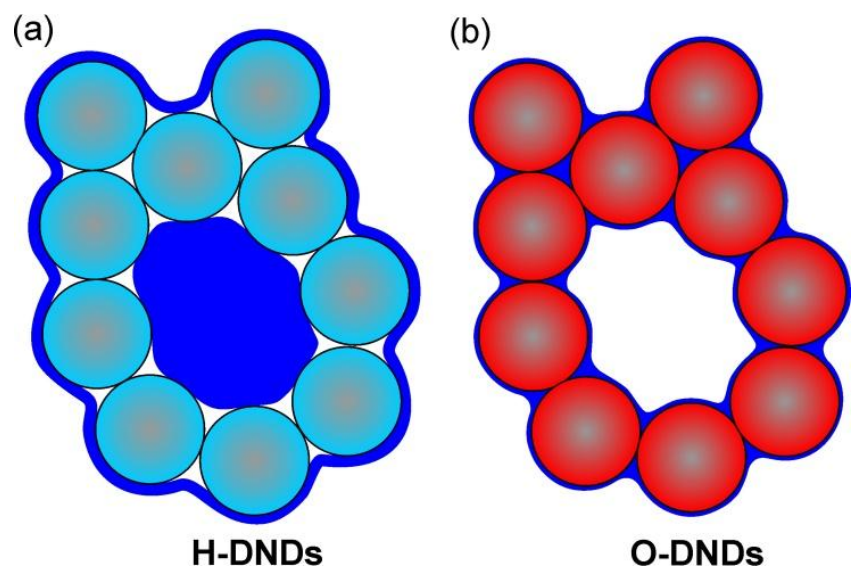

Figure 5. Simplified scheme of H-DND and O-DND interaction with water. The image (a) shows hydrophobic interaction resulting in formation of a thick, weakly bound water layer and possible water nanodroplet in the structure of H-DNDs. The image (b) shows hydrophilic interaction resulting in formation of a thin, strongly bound surface water layer on O-DNDs.

KPFM images (Figure 4) show that DNDs were deposited on HOPG in similar chain-like deposits using electrophoretic deposition ${ }^{19}$ without any obvious morphology dependence on the DNDs surface chemistry or water adsorption differences. The deposition of both H-DNDs and O-DNDs on HOPG from the related colloids took place preferentially at the step edges of otherwise atomically flat HOPG. This is most likely due to rather common effect of step edges that behave like low-energy sites and enable preferential deposition. Due to the equal morphology of the DND deposits a possible averaging effect, which may influence KPFM data when studied objects have close size to the tip ape ${ }^{38,39}$, is thus expected to be similar on both HDNDs and O-DNDs and the KPFM data are directly comparable.

The KPFM data show that the adsorbed water has a pronounced impact on electronic properties of DNDs, namely the CPD. The observed dependence of the CPD on the annealing temperature as well as differences related to the specific surface chemistry of DNDs can be explained by using the FTIR, TGA, DSC data and the corresponding model in Fig.5. The change of DNDs CPD is directly correlated with the removal of water adsorbed on DNDs as evidenced by the TGA and DSC data. In general, adsorbed water layers and ions may decrease the overall CPD contrast in KPFM under ambient conditions which is known as screening effect ${ }^{40}$. Our KPFM data indeed show a large shift of the CPD to more negative values during the annealing sequence for both H-DNDs and O-DNDs indicating strong screening effect at the room temperature. The screening effect is about 50\% stronger on H-DNDs than on O-DNDs. This may be readily explained by a thicker water layer as well as a different water structure on H-DNDs as suggested by FTIR and thermal analysis. However, we have to consider also a change of the electronic properties of diamond itself due to the removal of surface water.

As the Fermi level is pinned at the surface states of oxidized diamond ${ }^{41}$ we do not suppose other than water screening effects on the CPD of O-DNDs in the employed temperature 
range. In other words, the shift of the CPD to more negative values during the annealing sequence by as much as $350 \mathrm{mV}$ is caused only by gradual desorption of water. However, in the case of H-DNDs also the effect of transfer doping may influence the surface electronic properties including the work function ${ }^{42}$. The specific interaction of hydrogen terminated diamond with adsorbed ambient moisture leads to an electron transfer from diamond to the surface water layer resulting in a positive charge (holes) in the diamond and the formation of compensating counter anions in the water layer ${ }^{42,43}$. Based on thermal analysis it is reasonable to assume that the annealing up to $300^{\circ} \mathrm{C}$ leads to the complete desorption of water from both H-DNDs and ODNDs but whether the counter anions also desorb is unclear.

It was suggested by Ristein et al. that the desorption temperature of the counter anions from hydrogenated diamond in ultrahigh vacuum (UHV) is higher than $400^{\circ} \mathrm{C}^{42}$.. In accordance with the energetic band diagrams ${ }^{42}$, the removal of the counter anions should be accompanied by an abrupt decrease in the work function. In our case we did not observe any abrupt change of the H-DNDs work function but rather gradual change with increasing temperature as in the case of O-DNDs. In that respect note also that KPFM showed always a lower work function of H-DNDs compared to O-DNDs at both ambient and annealed condition, similarly to previous ambient KPFM studies ${ }^{10}$ and in agreement with the KPFM measurements on hydrogen and oxygen terminated bulk diamond ${ }^{44}$. Based on these arguments we assume that also in the case of $\mathrm{H}$ DNDs the effect of annealing on the KPFM data corresponds only to reduced screening via a gradual desorption of water from the H-DNDs. Nevertheless, this still needs to be corroborated by other techniques such as photoelectron spectroscopy ${ }^{45}$.

\section{Conclusion}

Thermal analysis (TGA/DSC), vibrational spectroscopy (FTIR) and Kelvin probe force microscopy were used to study the water interaction with the surface of hydrogenated and oxidized detonation nanodiamonds. By FTIR analysis of stretching and bending vibrations of adsorbed water molecules we identified a hydrophobic interaction of water with H-DNDs, in analogy with H-terminated bulk diamond. This leads to a higher amount of water on H-DNDs (4.4\%) compared to hydrophilic O-DNDs $(3.1 \%)$. At the same time, water binds more weakly to the H-DNDs surface and thus desorbs at lower temperatures $\left(\sim 250^{\circ} \mathrm{C}\right.$ on $\mathrm{H}$-DNDs, $\sim 300^{\circ} \mathrm{C}$ or higher on O-DNDs). This all indicated a different structure of water on hydrogenated and

oxidized DNDs as schematically shown by the presented model. By correlating FTIR, TGA/DSC and KPFM data we showed that the adsorbed water caused a significant screening effect (up to $0.5 \mathrm{~V}$ ) on the CPD of nanodiamonds which evolved gradually with the annealing temperature up to $300^{\circ} \mathrm{C}$. Change of the surface Fermi level (transfer doping, surface state pinning) can be neglected in this annealing range. The screening effect is more pronounced on H-DNDs, which can be attributed to the different water amount and structure as provided by the FTIR and thermal analysis.

\section{Acknowledgement}


S. Stehlik acknowledges the SCIEX project 13.154 for financial support. B. Rezek acknowledges the GACR project 15-01809S. This work was also partly supported by the Swiss National Science Foundation and the Commission for Technology and Innovation CTI (project 16039.1 PFNM-NM)

\section{References}

${ }^{1}$ [1] V. Pichot, M. Comet, B. Risse, D. Spitzer, Detonation of nanosized explosive: New mechanistic model for nanodiamond formation, Diamond and Related Materials. 54 (2015) 5963. doi:10.1016/j.diamond.2014.09.013.

2 [2] V. Pichot, B. Risse, F. Schnell, J. Mory, D. Spitzer, Understanding ultrafine nanodiamond formation using nanostructured explosives, Scientific Reports. 3 (2013). doi:10.1038/srep02159.

3 [3] O.A. Shenderova, V.V. Zhirnov, D.W. Brenner, Carbon Nanostructures, Critical Reviews in Solid State and Materials Sciences. 27 (2002) 227-356. doi:10.1080/10408430208500497.

${ }^{4}$ [4] V.N. Mochalin, O. Shenderova, D. Ho, Y. Gogotsi, The properties and applications of nanodiamonds, Nature Nanotechnology. 7 (2011) 11-23. doi:10.1038/nnano.2011.209.

${ }^{5}$ [5] A. Krueger, D. Lang, Functionality is Key: Recent Progress in the Surface Modification of Nanodiamond, Advanced Functional Materials. 22 (2012) 890-906.

doi:10.1002/adfm.201102670.

${ }^{6}$ [6] T. Ando, S. Inoue, M. Ishii, M. Kamo, Y. Sato, O. Yamada, et al., Fourier-transform infrared photoacoustic studies of hydrogenated diamond surfaces, J. Chem. Soc., Faraday Trans. 89 (1993) 749-751.

7 [7] H.A. Girard, E. Scorsone, S. Saada, C. Gesset, J.C. Arnault, S. Perruchas, et al., Electrostatic grafting of diamond nanoparticles towards 3D diamond nanostructures, Diamond and Related Materials. 23 (2012) 83-87. doi:10.1016/j.diamond.2012.01.021.

${ }^{8}$ [8] T. Jiang, K. Xu, S. Ji, FTIR studies on the spectral changes of the surface functional groups of ultradispersed diamond powder synthesized by explosive detonation after treatment in hydrogen, nitrogen, methane and air at different temperatures, J. Chem. Soc., Faraday Trans. 92 (1996) 3401-3406.

${ }^{9}$ [9] O.A. Williams, J. Hees, C. Dieker, W. Jäger, L. Kirste, C.E. Nebel, Size-Dependent Reactivity of Diamond Nanoparticles, ACS Nano. 4 (2010) 4824-4830. doi:10.1021/nn100748k. ${ }^{10}$ [10] S. Stehlik, T. Petit, H.A. Girard, J.-C. Arnault, A. Kromka, B. Rezek, Nanoparticles Assume Electrical Potential According to Substrate, Size, and Surface Termination, Langmuir. 29 (2013) 1634-1641. doi:10.1021/la304472w.

${ }^{11}$ [11] S. Osswald, G. Yushin, V. Mochalin, S.O. Kucheyev, Y. Gogotsi, Control of sp ${ }^{2} / \mathrm{sp}^{3}$ Carbon Ratio and Surface Chemistry of Nanodiamond Powders by Selective Oxidation in Air, Journal of the American Chemical Society. 128 (2006) 11635-11642. doi:10.1021/ja063303n. 12 [12] V. Pichot, M. Comet, E. Fousson, C. Baras, A. Senger, F. Le Normand, et al., An efficient purification method for detonation nanodiamonds, Diamond and Related Materials. 17 (2008) 13-22. doi:10.1016/j.diamond.2007.09.011.

${ }^{13}$ [13] J. Hees, A. Kriele, O.A. Williams, Electrostatic self-assembly of diamond nanoparticles, Chemical Physics Letters. 509 (2011) 12-15. doi:10.1016/j.cplett.2011.04.083.

${ }^{14}$ [14] A. Bolker, C. Saguy, R. Kalish, Transfer doping of single isolated nanodiamonds, studied by scanning probe microscopy techniques, Nanotechnology. 25 (2014) 385702.

doi:10.1088/0957-4484/25/38/385702. 
${ }^{15}$ [15] T. Kondo, I. Neitzel, V.N. Mochalin, J. Urai, M. Yuasa, Y. Gogotsi, Electrical conductivity of thermally hydrogenated nanodiamond powders, Journal of Applied Physics. 113 (2013) 214307. doi:10.1063/1.4809549.

${ }^{16}$ [16] M.V. Korobov, N.V. Avramenko, A.G. Bogachev, N.N. Rozhkova, E. Osawa, Nanophase of Water in Nano-Diamond Gel, Journal of Physical Chemistry C. 111 (2007) 7330-7334. doi:10.1021/jp0683420.

${ }^{17}$ [17] T. Petit, H. Yuzawa, M. Nagasaka, R. Yamanoi, E. Osawa, N. Kosugi, et al., Probing Interfacial Water on Nanodiamonds in Colloidal Dispersion, The Journal of Physical Chemistry Letters. 6 (2015) 2909-2912. doi:10.1021/acs.jpclett.5b00820.

18 [18] S.S. Batsanov, S.M. Gavrilkin, A.S. Batsanov, K.B. Poyarkov, I.I. Kulakova, D.W. Johnson, et al., Giant dielectric permittivity of detonation-produced nanodiamond is caused by water, Journal of Materials Chemistry. 22 (2012) 11166. doi:10.1039/c2jm30836c.

19 [19] L. Schmidlin, V. Pichot, S. Josset, R. Pawlak, T. Glatzel, S. Kawai, et al., Twodimensional nanodiamond monolayers deposited by combined ultracentrifugation and electrophoresis techniques, Applied Physics Letters. 101 (2012) 253111. doi:10.1063/1.4772983. ${ }^{20}$ [20] C. Sommerhalter, T.W. Matthes, T. Glatzel, A. Jäger-Waldau, M.C. Lux-Steiner, Highsensitivity quantitative Kelvin probe microscopy by noncontact ultra-high-vacuum atomic force microscopy, Applied Physics Letters. 75 (1999) 286. doi:10.1063/1.124357.

${ }^{21}$ [21] H. Diesinger, D. Deresmes, J.-P. Nys, T. Mélin, Kelvin force microscopy at the second cantilever resonance: An out-of-vacuum crosstalk compensation setup, Ultramicroscopy. 108 (2008) 773-781. doi:10.1016/j.ultramic.2008.01.003.

22 [22] S. Ji, T. Jiang, K. Xu, S. Li, FTIR study of the adsorption of water on UDD surface, Applied Surface Science 133 (1998) 231-238.

23 [23] G. Socrates, Infrared and Raman characteristic group frequencies: tables and charts. (2001), 3rd ed., Wiley, Chichester

${ }^{24}$ [24] S. Stehlik, M. Varga, M. Ledinsky, V. Jirasek, A. Artemenko, H. Kozak, et al., Size and Purity Control of HPHT Nanodiamonds down to $1 \mathrm{~nm}$, The Journal of Physical Chemistry C. (2015) 150825003821009. doi:10.1021/acs.jpcc.5b05259.

${ }^{25}$ [25] N. Kitadai, T. Sawai, R. Tonoue, S. Nakashima, M. Katsura, K. Fukushi, Effects of Ions on the OH Stretching Band of Water as Revealed by ATR-IR Spectroscopy, Journal of Solution Chemistry. 43 (2014) 1055-1077. doi:10.1007/s10953-014-0193-0.

${ }^{26}$ [26] M. Praprotnik, D. Janežič, J. Mavri, Temperature Dependence of Water Vibrational Spectrum: A Molecular Dynamics Simulation Study, The Journal of Physical Chemistry A. 108 (2004) 11056-11062. doi:10.1021/jp046158d.

27 [27] L. F. Scatena, M. G. Brown, G. L. Richmond, Water at hydrophobic surfaces: weak hydrogen bonding and strong orientation effects, Science 292 (2001) 908-912.

doi:10.1126/science.1059514.

28 [28] K. Larsson, J. Ristein, Diamond Surface Conductivity under Atmospheric Conditions: Theoretical Approach, The Journal of Physical Chemistry B. 109 (2005) 10304-10311. doi:10.1021/jp050419h.

${ }^{29}$ [29] D. Eisenberg, W. Kauzmann, The Structure and Properties of Water (Oxford Univ. Press, New York, 1969).

30 [30] J.G. Davis, B.M. Rankin, K.P. Gierszal, D. Ben-Amotz, On the cooperative formation of non-hydrogen-bonded water at molecular hydrophobic interfaces, Nature Chemistry. 5 (2013) 796-802. doi:10.1038/nchem.1716. 
31 [31] V. Mochalin, S. Osswald, Y. Gogotsi, Contribution of Functional Groups to the Raman Spectrum of Nanodiamond Powders, Chemistry of Materials. 21 (2009) 273-279. doi:10.1021/cm802057q.

32 [32] A.E. Aleksenskii, M.V. Baidakova, A.Y. Vul, V.I. Siklitskii, The structure of diamond nanoclusters, Physics of the Solid State. 41 (1999) 668-671.

33 [33] O.V. Tomchuk, D.S. Volkov, L.A. Bulavin, A.V. Rogachev, M.A. Proskurnin, M.V. Korobov, et al., Structural Characteristics of Aqueous Dispersions of Detonation Nanodiamond and Their Aggregate Fractions as Revealed by Small-Angle Neutron Scattering, The Journal of Physical Chemistry C. 119 (2015) 794-802. doi:10.1021/jp510151b.

34 [34] J.C. Eriksson, U. Henriksson, Thermodynamic Properties of Bridging Clusters in Thin Films of Water between Hydrophobic Surfaces Assessed from Surface Force Isotherms, Langmuir. 29 (2013) 4789-4795. doi:10.1021/la400446p.

35 [35] R. Sappok, H. Boehm, Surface chemistry of diamond - II. Formation, properties and structure of the surface oxides, Carbon 6, (1968) 573.

${ }^{36}$ [33] E.T. Herruzo, H. Asakawa, T. Fukuma, R. Garcia, Three-dimensional quantitative force maps in liquid with 10 piconewton, angstrom and sub-minute resolutions, Nanoscale. 5 (2013) 2678-2685. doi:10.1039/C2NR33051B.

${ }^{37}$ [37] F.K. de Theije, M.F. Reedijk, J. Arsic, W.J.P. van Enckevort, E. Vlieg, Atomic structure of diamond $\{111\}$ surfaces etched in oxygen water vapor, Physical Review B. 64 (2001). doi:10.1103/PhysRevB.64.085403.

38 [38] H.O. Jacobs, P. Leuchtmann, O.J. Homan, A. Stemmer, Resolution and contrast in Kelvin probe force microscopy, Journal of Applied Physics. 84 (1998) 1168-1173.

H.O. Jacobs, P. Leuchtmann, O.J. Homan, A. Stemmer, Journal of Applied Physics 84 (1998) $1168-1173$.

${ }^{39}$ [39] T. Glatzel, S. Sadewasser, M.C. Lux-Steiner, Amplitude or frequency modulationdetection in Kelvin probe force microscopy, Applied Surface Science. 210 (2003) 84-89. doi:10.1016/S0169-4332(02)01484-8.

${ }^{40}$ [40] H. Sugimura, Y. Ishida, K. Hayashi, O. Takai, N. Nakagiri, Potential shielding by the surface water layer in Kelvin probe force microscopy, Applied Physics Letters. 80 (2002) 1459. doi:10.1063/1.1455145.

${ }^{41}$ [41] F. Maier, J. Ristein, L. Ley, Electron affinity of plasma-hydrogenated and chemically oxidized diamond (100) surfaces, Physical Review B. 64 (2001).

doi:10.1103/PhysRevB.64.165411.

42 [42] F. Maier, M. Riedel, B. Mantel, J. Ristein, L. Ley, Origin of surface conductivity in diamond, Physical Review Letters. 85 (2000) 3472.

43 [43] V. Chakrapani, J.C. Angus, A.B. Anderson, S.D. Wolter, B.R. Stoner, G.U. Sumanasekera, Charge Transfer Equilibria Between Diamond and an Aqueous Oxygen Electrochemical Redox Couple, Science. 318 (2007) 1424-1430. doi:10.1126/science.1148841. 44 [44] B. Rezek, C.E. Nebel, Kelvin force microscopy on diamond surfaces and devices, Diamond and Related Materials. 14 (2005) 466-469. doi:10.1016/j.diamond.2005.01.041. 45 [45] T. Petit, H.A. Girard, A. Trouvé, I. Batonneau-Gener, P. Bergonzo, J.-C. Arnault, Surface transfer doping can mediate both colloidal stability and self-assembly of nanodiamonds, Nanoscale. 5 (2013) 8958. doi:10.1039/c3nr02492j. 